\title{
Evaluation of Diagnostic Tests for Visceral Leishmaniasis in Iran: A Meta- Analysis
}

\author{
Azar Najafi ${ }^{1,2}$, Entezar Abbasi Somar ${ }^{1,2}$, Kourosh Sayehmiri $^{3}$, Hamidreza Majidiani ${ }^{4}$, Asad Mirzaei $^{2}$ \\ ${ }^{1}$ Department of Medical Parasitology, Paramedical Faculty, Ilam University of Medical Sciences, Ilam, Iran \\ ${ }^{2}$ Student Research Committee, Ilam University of Medical Sciences, Ilam, Iran \\ ${ }^{3}$ Department of Biostatistics, Psychosocial injuries Research center, Ilam University of Medical Sciences, Ilam, Iran \\ ${ }^{4}$ Department of Parasitology, Faculty of Medical Sciences, Tarbiat Modares University, Tehran, Iran
}

\begin{abstract}
Objective: There exist various diagnostic tests to detect visceral leishmaniasis, but the most efficient ones have not been demonstrated yet. Herein, we evaluated the accuracy of these diagnostic tests in Iran.

Methodology: English and Persian database searching was done from 1993 to 2016. According to heterogeneity of the studies, estimations of the study were pooled by random-effects model. Totally, twenty investigations were eligible for current review with 2115 patients as pooled sample size.
\end{abstract}

Results: The overall pooled estimate of sensitivity and specificity for all diagnostic tests were $0.92([95 \% \mathrm{Cl}=0.90$ $0.95])$ and $0.92([95 \% \mathrm{Cl}=0.89-0.94])$, respectively. Also, the separate sensitivity and specificity of each test were as follow: PCR: 1.00 ([95\% Cl = 0.99-1.01]) and $0.37([95 \% \mathrm{Cl}=0.25-0.49])$; DAT: $0.93([95 \% \mathrm{Cl}=0.89-0.96])$ and $0.92([95 \% \mathrm{Cl}=0.89-0.94])$; ELISA: $0.78([95 \% \mathrm{Cl}=0.47-1.09])$ and $1.00([95 \% \mathrm{Cl}=0.99-1.01])$ as well as IFA: $0.50([95 \% \mathrm{Cl}=0.29-0.72])$ and $0.96([95 \% \mathrm{Cl}=0.95-0.97])$.

Conclusions: The specificity and sensitivity of PCR and DAT as molecular and serological tests, respectively, are remarkable to detect visceral leishmaniasis; hence, it is highly recommended to simultaneously employ these diagnostic tests for this widespread infection. J Microbiol Infect Dis 2019; 9(1): 16-22.

Keywords: Visceral Leishmaniasis, sensitivity, specificity, diagnostic tests, L. infantum, Iran

\section{INTRODUCTION}

Visceral leishmaniasis (VL) or kala-azar is a zoonotic, neglected parasitic disease caused by Leishmania spp., with Phlebotomus and Lutzomyia sanflies as primary vectors [1]. The $V L$ nomenclature includes those species in Leishmania donovani complex, comprising $L$. infantum, L. donovani and L. chagasi [2]. More than one hundred countries suffer from VL as an endemic disease, predominantly Brazil, Ethiopia, Nepal, India, Kenya, Somalia and Sudan [3]. Annually, the disease affects 200,000 to 400,000 individuals, and if untreated, it would be fatal particularly in endemic areas, with an average of 30,000 deaths [4]. In Iran, the Mediterranean type of kala-azar is dominated in seven wide regions [4,5], which is caused by $L$. infantum $[6,7]$ with estimated incidence rate of 100-300 cases/year [8]. Domestic dogs, foxes and jackals play a major role as reservoir hosts for the disease and Phlebotomus major is the predominant vector in Iran $[5,9]$. The kala-azar clinical pictures are including fever, hepatosplenomegaly, anaemia, haemorrhagic manifestations, cardiac abnormalities, jaundice, diarrhea, hypersplenomegaly, hyperalbuminemia and pancytopenia [10], which may be mistaken with malaria, typhoid and tuberculosis; this issue would complicate appropriate diagnosis of VL [11]. Delay in the diagnosis of the infection in patients is associated with high mortality especially in children [11,12]. Hence, devising an easy, rapid and non-invasive diagnostic test for accurate detection of $\mathrm{VL}$ is an important step in prevention strategies [13,14]. Culturing spleen aspirates in order to probe the parasite is the gold standard method for VL diagnosis. However, due to its invasive nature, lymph node 
and bone marrow aspirates are alternatively used. Conventional serological tests such as enzyme-linked immunosorbent assay (ELISA), indirect hemagglutination test (IHA), immunochromatographic tests (ICTs), direct agglutination test (DAT) and countercurrent immunoelectrophoresis (CCIE) possess favorable sensitivity and specificity and lack cross-reactivity, so they can be considered as a good alternative for traditional parasitological methods such as direct smear, culture method and animal inoculation [11,14]. Different clinical manifestations of $V L$ on the one hand and wide array of diagnostic methods on the other hand implicate an in-depth analysis of sensitivity and specificity of detection methods to get closer to a gold standard test and reduce treatment costs. This meta-analysis would shed light on the performance and accuracy of exerted diagnostic tests for canine and human visceral leishmaniasis in Iran.

\section{METHODS}

\section{Search strategy}

The systematic search for the accuracy of diagnostic tests of kala-azar in Iran was done by screening online released literature both in English and Persian languages. Totally, seven English databases (Scopus, PubMed, Science Direct, ProQuest, Web of Science, Springer and Google Scholar) and four Persian databases (scientific information database (SID), Iran Doc, Iran Medex and Magiran) were probed for published articles from August 1993 - June 2016. Several medical subject heading (MeSH) terms were used for searching procedure, including: "visceral leishmaniasis", "sensitivity", "specificity", "kala-azar" and "diagnostic tests". The equivalent of these terms was used to search Persian databases.

\section{Inclusion and exclusion criteria}

Based on the descriptions by world health organization, sensitivity is the ability of a test to detect the disease, while specificity is the ability of a test to reveal healthy individuals [15). Related topics obtained during initial database searching were saved and their abstracts were recorded in a word file for further assessment. Subsequently, after primary screening, the full texts of potentially eligible papers were downloaded and carefully read by two independent reviewers ( $A N$ and $A M$ ) to check eligibility. One author (AA) extracted the requisite information and a second author (AM) rechecked them. The inclusion criteria were including: 1) all study types except of review articles; 2) with the subject of the prevalence or diagnosing visceral leishmaniasis in human and animal hosts; 3) using serological or molecular methods excluding traditional parasitological examinations; 4) with evaluation of sensitivity and specificity of diagnostic tests; 5) having determined sample size and exact number of infected cases. A data extraction form was used to gather information from selected papers (Table 1). Present survey was conducted relied on the PRISMA guideline (preferred reporting items for systematic reviews and meta-analyses) [16].

\subsection{Meta-analysis}

The model of random effects was used to estimate the sensitivity and specificity of each study. The results of meta-analysis were depicted as a forest plot, indicating the estimates of sensitivity and specificity, summary measure and confidence interval of every record. Also, Cochran's Q statistic and 12 index were done to evaluate heterogeneity and inconsistency, respectively. To assess the relationship between sensitivity and specificity with year of publications meta-regression was used. All analysis was carried out with SPSS statistical software.

\section{RESULTS}

Initially, a number of 150 investigations were gathered during database exploration. Of these, only 20 studies exactly met the inclusion criteria in this study, as depicted in a flowchart (Fig 1). The properties of included records are shown in supplementary Table 1. Different detection methods were found to be used for VL diagnosis in Iran, consisting of: DAT (10 studies), ELISA (3 studies), Polymerase Chain Reaction (PCR) (3 studies) and IFAT (2 studies). Table 2 represents the estimates of the accuracy of VL diagnostics and their respective $95 \%$ confidence intervals $(\mathrm{Cl})$. The total estimations for sensitivity and specificity were $0.92([95 \% \mathrm{Cl}=0.90-0.95])$ and 0.92 ([95\% Cl=0.89-0.94]), respectively (Figures 2 and 3 ). 
Table 1. Characteristics of included studies in the meta-analysis.

\begin{tabular}{|c|c|c|c|c|c|c|c|c|c|c|c|}
\hline ID & Author & Year & $\begin{array}{r}\text { Study } \\
\text { Type }\end{array}$ & GA & $\begin{array}{l}\text { Study } \\
\text { City }\end{array}$ & EH & $\begin{array}{c}\text { Diagnostic } \\
\text { test }\end{array}$ & $\begin{array}{c}\text { No. of } \\
\text { Samples }\end{array}$ & $\begin{array}{c}\text { No. of } \\
\text { Positive }\end{array}$ & Sample & Ref. \\
\hline 1 & Khademvatan & 2011 & DE & South & Azerbaijan & Human & DAT & 385 & 71 & $\begin{array}{l}\text { Urine, } \\
\text { blood }\end{array}$ & 12 \\
\hline $1-2$ & Khademvatan & 2011 & DE & South & $\begin{array}{c}\text { East } \\
\text { Azerbaijan }\end{array}$ & Human & ELISA & 385 & 91 & $\begin{array}{l}\text { Urine, } \\
\text { blood }\end{array}$ & 12 \\
\hline $1-3$ & Khademvatan & 2011 & DE & South & $\begin{array}{c}\text { East } \\
\text { Azerbaijan }\end{array}$ & Human & IFA & 385 & 60 & $\begin{array}{l}\text { Urine, } \\
\text { blood }\end{array}$ & 12 \\
\hline 4 & Mohammadi & 2011 & $\mathrm{CC}$ & South & $\begin{array}{l}\text { Meshkin } \\
\text { Shahr }\end{array}$ & Animal & ELISA & 60 & 7 & Serum & 25 \\
\hline $4-5$ & Mohammadiha & 2013 & $\mathrm{CC}$ & South & $\begin{array}{l}\text { Meshkin } \\
\text { Shahr }\end{array}$ & Human & DAT & 200 & 77 & Serum & 19 \\
\hline $4-6$ & Mohammadiha & 2013 & $\mathrm{CC}$ & South & $\begin{array}{l}\text { Meshkin } \\
\text { Shahr }\end{array}$ & Human & PCR & 200 & 77 & Blood & 19 \\
\hline $4-7$ & Mohammadiha & 2013 & $\mathrm{CC}$ & South & $\begin{array}{l}\text { Meshkin } \\
\text { Shahr }\end{array}$ & Animal & DAT & 200 & 72 & Serum & 19 \\
\hline $4-8$ & Mohammadiha & 2013 & $\mathrm{CC}$ & South & $\begin{array}{l}\text { Meshkin } \\
\text { Shahr }\end{array}$ & Animal & PCR & 200 & 72 & Blood & 19 \\
\hline $4-9$ & Mohammadiha & 2013 & $\mathrm{CC}$ & Northeast & $\begin{array}{l}\text { Meshkin } \\
\text { Shahr }\end{array}$ & Animal & DAT & 167 & 149 & Serum & 19 \\
\hline $\begin{array}{l}4- \\
10\end{array}$ & Mohammadiha & 2103 & $\mathrm{CC}$ & Northeast & $\begin{array}{l}\text { Meshkin } \\
\text { Shahr }\end{array}$ & Animal & PCR & 167 & 104 & Blood & 19 \\
\hline 11 & Mahami & 2008 & CS & South & Ardebil & Human & DAT & 1155 & 32 & Serum & 26 \\
\hline $\begin{array}{l}11- \\
12\end{array}$ & Mahami & 2008 & C & & Ardebil & Human & ELISA & 1155 & 8 & Serum & 26 \\
\hline $\begin{array}{l}11- \\
13\end{array}$ & Mahami & 2008 & CS & South & Ardebil & Human & IFA & 1155 & 32 & Serum & 26 \\
\hline 14 & Mazloumi & 2002 & $\mathrm{CC}$ & South & Ardebil & Human & DAT & 3872 & 271 & Serum & 24 \\
\hline $\begin{array}{l}14- \\
15 \\
\end{array}$ & Mazloumi & 2002 & $\mathrm{CC}$ & South & Ardebil & Animal & DAT & 199 & 43 & Serum & 24 \\
\hline
\end{tabular}

$\mathrm{ID}=$ No. of Article, $\mathrm{DE}=$ Descriptive Epidemiology, $\mathrm{CC}=$ Case Control, $\mathrm{GA}=$ Geographic area $\mathrm{S}=$ Cross sectional, EH=Examined Host

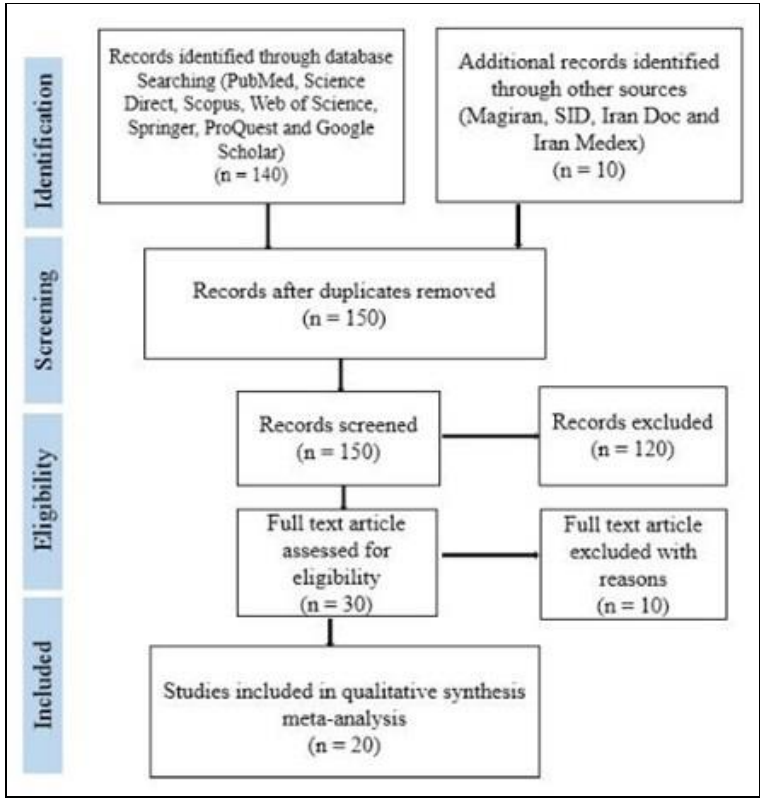

Figure 1. Flow diagram describing the study design process.

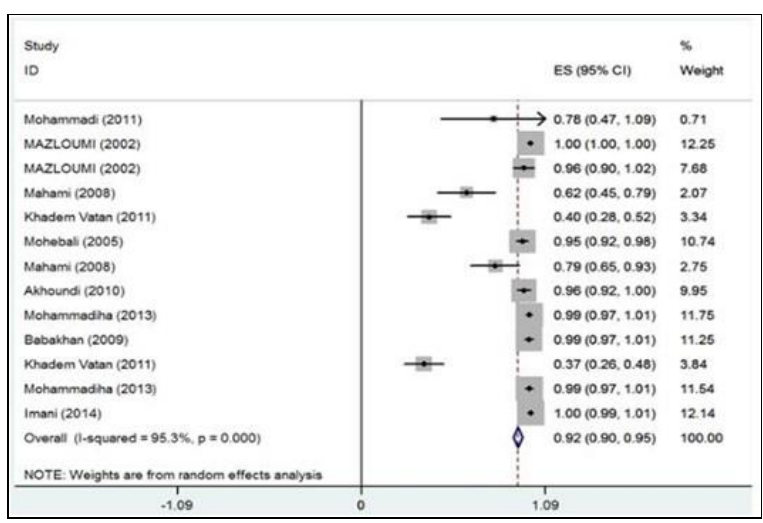

Figure 2. The overall estimate of sensitivity of diagnostic tests based on the random effects model, the midpoint of each line segments estimation of the lozenge Estimates of sensitivity (ES) and length the line segments (95\% confidence intervals) study shows each ES lozenge Mark ES for all studies show. 


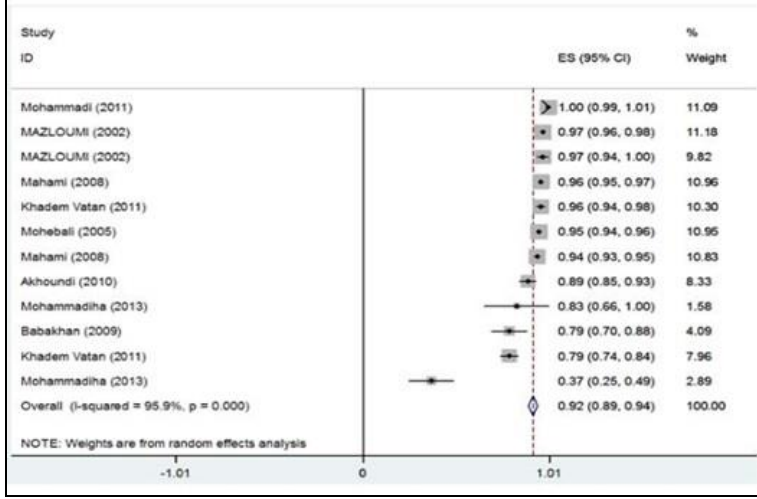

Figure 3. The overall estimate of specificity of diagnostic tests based on the random effects model, the midpoint of each line segments estimation of the lozenge Estimates of specificity (ES) and length the line segments (95\% confidence intervals) study shows each ES lozenge Mark ES for all studies show.

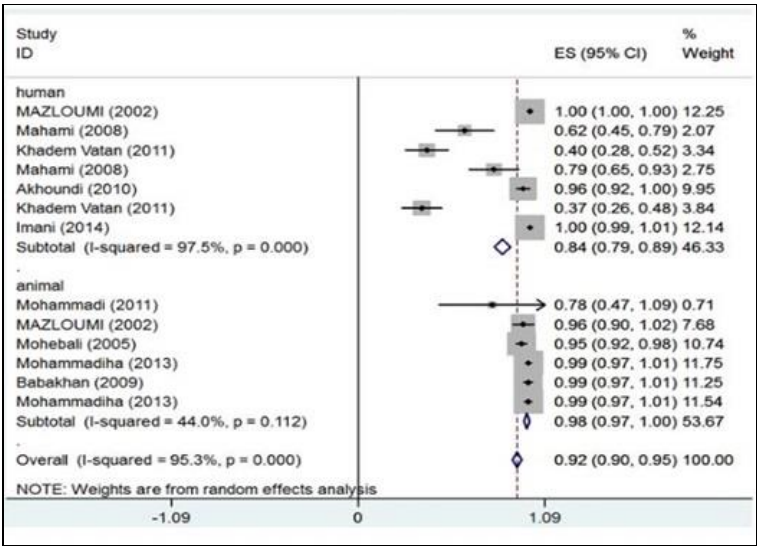

Figure 4. Estimates of sensitivity of diagnostic tests in humans and animals based on the random effects model.

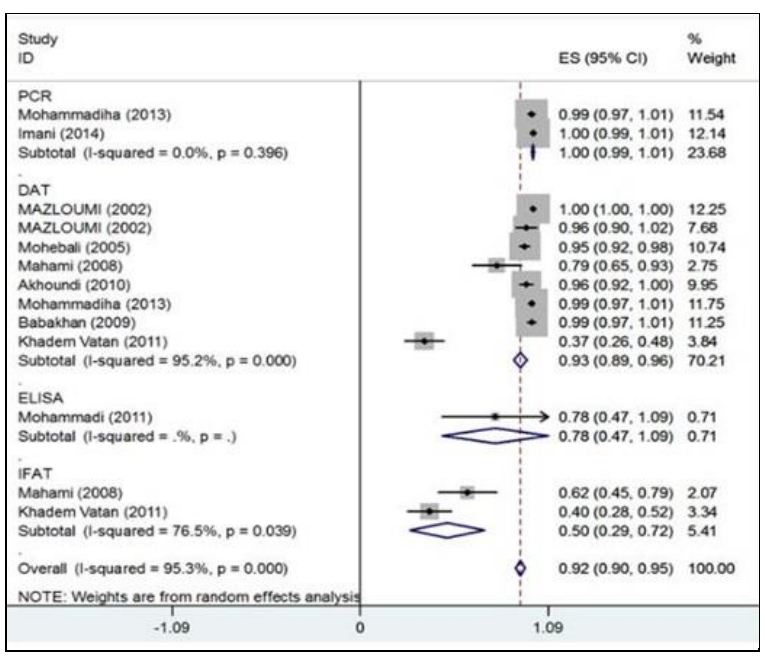

Figure 5. Estimates of specificity of diagnostic tests in humans and animals based on the random effects model.

According to the results, the sensitivity and specificity of the tests in humans are 0.84 ([95\% $\mathrm{Cl}=0.79-0.89])$ and $0.93([95 \% \mathrm{Cl}=0.90-$ $0.95])$, respectively. In case of animals, the sensitivity was calculated as $0.98([95 \% \mathrm{Cl}=$ 0.97-1.00]), while the specificity was estimated to be 0.87 ([95\% Cl =0.81-0.93]) (Figs 4, 5). The pooled estimates of sensitivity of VL tests in various geographic areas of Iran was 0.97 ([95\% $\mathrm{Cl}=0.94-1.01])($ Fig 6)

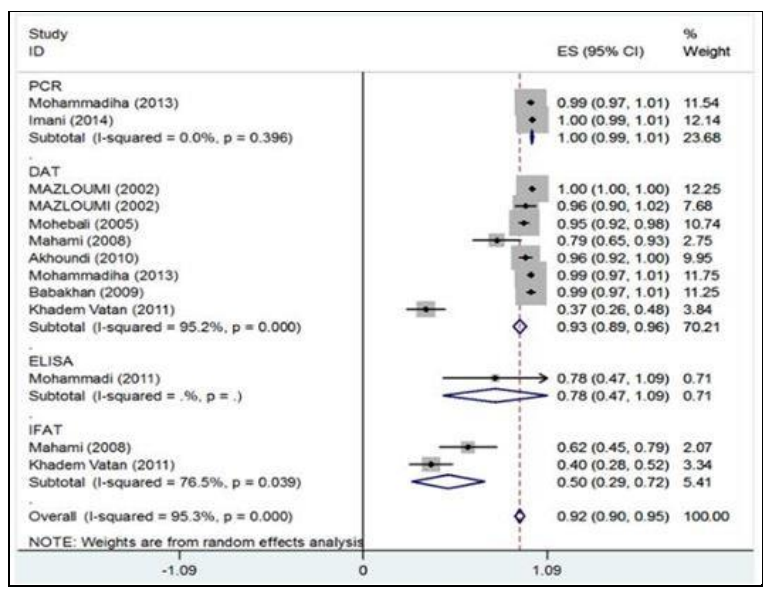

Figure 6. Estimates of sensitivity of diagnostic tests for (PCR, DAT, ELISA and IFAT) based on the random effects model.

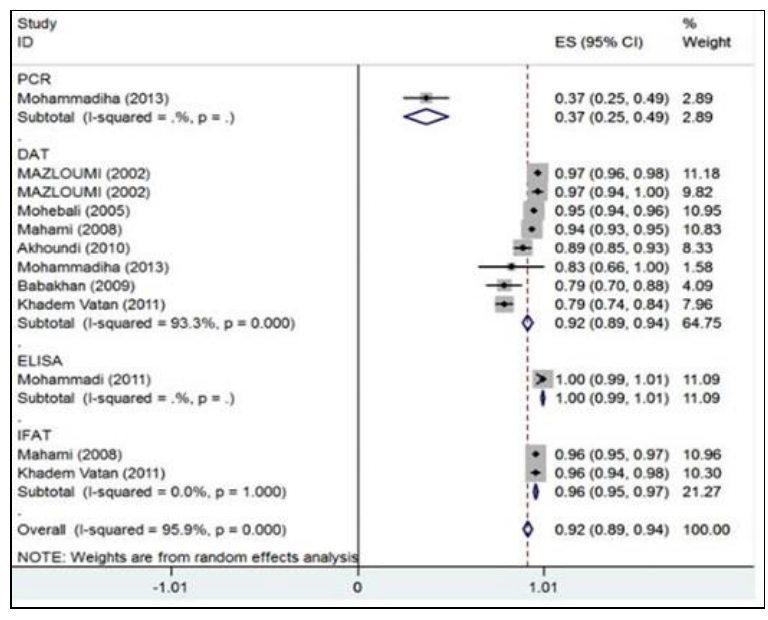

Figure 7. Estimates of specificity of diagnostic tests for (PCR, DAT, ELISA and IFAT) based on the random effects model. 
The highest sensitivities were dedicated to PCR with $1.00([95 \% \mathrm{Cl}=0.99-1.01])$ followed by DAT with 0.93 ([95\% Cl=0.89-0.96]), ELISA with 0.78 $([95 \% \mathrm{Cl}=0.47-1.09])$ and IFAT with 0.50 ([95\% $\mathrm{Cl}=0.29-0.72])$. In case of specificity, PCR is pioneer with 0.37 ([95\% Cl=0.25-0.49]), followed by DAT with $0.92([95 \% \mathrm{Cl}=0.89-0.94])$ and ELISA with $1.00([95 \% \mathrm{Cl}=0.99-1.01])$ (Fig 7). Based on meta-regression findings, there was not observed any changes between the overall estimates of sensitivity and specificity with year of publications (Figs 8, 9).

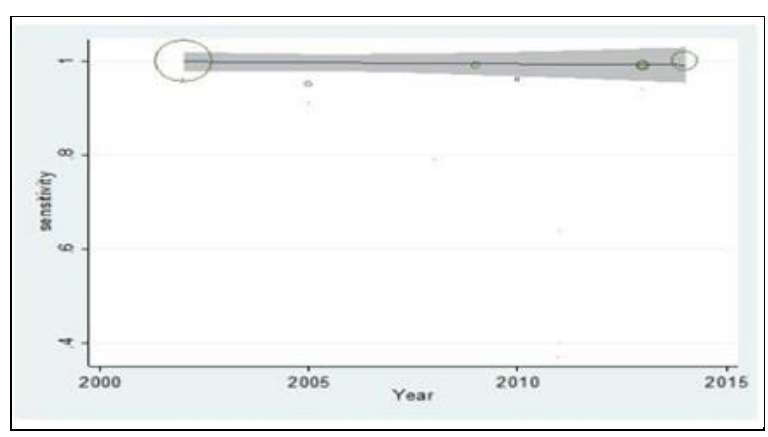

Figure 8. Meta-regression charts of the sensitivity of diagnostic tests based on year of studies and sample size.

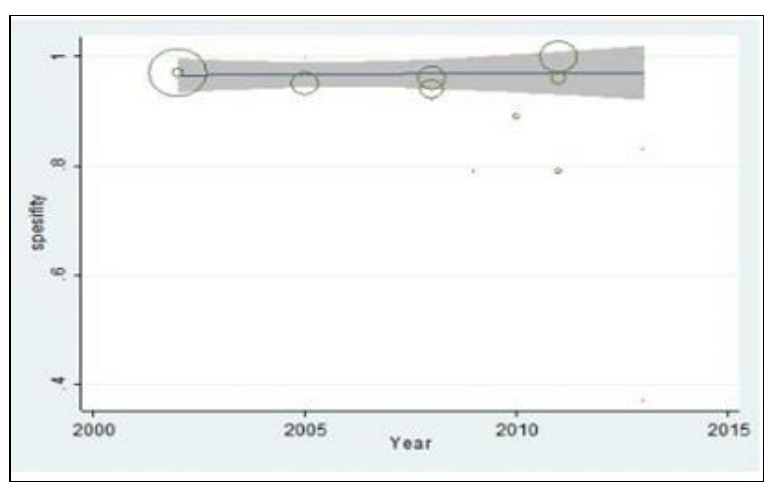

Figure 9. Meta-regression chart of the specificity of diagnostic tests based on year of studies and sample size.

\section{DISCUSSION}

Current meta-analysis deals with the appraisal of the accuracy of VL diagnostic methods in human and animal populations of Iran during last two decades. Since common parasitological detection methods for kala-azar, encompassing bone marrow aspirates or biopsy, lack appropriate sensitivity, are invasive and crossreact with other infections, only serological and molecular diagnostic tests were taken into account in this review $[17,18]$.

Regarding the fatal nature of infection and high toxicity of treatments, the progressive need to accurate molecular diagnostics, particularly in immunocompromised patients where serological tests doesn't provide good results as well as unable to discriminate relapse cases, is inevitable. Based on our findings, high sensitivity and specificity was proved for DAT and PCR (100\% and $95 \%$ for sensitivity and $95 \%$ and $93 \%$ for specificity, respectively). Investigations have reported different ranges of specificity and sensitivity for PCR in VL-infected hosts. For instance, the sensitivity and specificity of molecular diagnosis for canine VL in northwest of Iran were $98 \%$ and $88.2 \%$, respectively [19], whereas $69 \%$ sensitivity was recorded for PCR isolation of Leishmania DNA from bone marrow slides in Nepal. These differences are probably due to the type of primer oligonucleotides, parasite species and level of parasitemia [20].In a prevalence investigation on $67 \mathrm{VL}$-suspected patients by PCR and IFA in Shiraz, 23 cases were positive using PCR method with $82.5 \%$ and $40 \%$ sensitivity and specificity, respectively. [21]. Our findings demonstrated that the highest sensitivity and specificity are associated with DAT with $92 \%$ and 93\%, respectively. Accordingly, in a study in Brazil it was shown that DAT sero-diagnosis is a very accurate technique for VL with $97.8 \%$ sensitivity and $100 \%$ specificity [22]. Furthermore, the results of a systematic review in Iran represented that DAT have a sensitivity and specifitcity about 94.8 and 85.9, respectively, consistent with our results [23]. Several studies on the diagnostic tests of VL in animals and humans demonstrated that mostly animal populations were exposed to the infection, especially owing to the likely role of animals, especially canines, as parasite reservoirs in rural mountainous endemic regions $[9,24]$. Consequently, health officials should pay special attention to dogs as common reservoir hosts in endemic regions to better perform preventive measures.

Current review assessed the accuracy of exerted diagnostic tests for $\mathrm{VL}$ in Iran, and represented that there is a high degree of sensitivity and specificity for PCR on bone marrow and blood samples. No statistically significant difference was found between the 
performance of PCR on serum and bone marrow aspirates; hence, it could be inferred that only blood sample is adequate for the diagnosis. Also, it was revealed that the sensitivity of PCR on serum and bone marrow samples was remarkable $(0.99(95 \% \mathrm{Cl}=0.97$ 1.1) vs $1.00(95 \% \mathrm{Cl}=0.99-1.1))$, while the specificity was very low $(0.37(95 \% \mathrm{Cl}=0.25$ $0.49)$ ). Methodologically, it is anticipated that negative hosts in case-control studies be actually healthy, but it has been frequently observed that these individuals are suspects. High number of PCR positive persons in suspected cases showing a false-negative reference is justified by a group of false positive individuals as true $\mathrm{VL}$ cases, when considering that gold standard test is inefficient and PCR sensitivity is premier than the gold standard. Moreover, a significant number of VL carriers in endemic areas that manifest splenomegaly and chronic fever may be detected as false positive in VL PCR experiments. Hence, PCR positivity may be interpreted as infection index not a disease marker. Furthermore, with respect to the low, improper immune responses in immunosuppressed individuals such as HIV patients, PCR is taken into account as a promising alternative with high sensitivity for serodiagnosis of $\mathrm{VL}$ in these at-risk populations.

Our study was confined to some limitations: 1) lack of sufficient studies on the comparison of VL diagnostic tests, 2) lack of sensitivity and specificity appraisal in some studies, and 3) absence of a gold standard test to compare sensitivity and specificity of other tests with. These limitations may be vital for the precise evaluation of diagnostic tests of visceral leishmaniasis in Iran.

$\mathrm{PCR}$ is an accurate molecular technique for the amplification of small amounts of parasite DNA with high sensitivity and specificity, particularly in immunocompromised patients, children and individuals with early stages of the disease. Although PCR may result in false positive outcomes among examined populations. In comparison to PCR, ELISA and IFA, which require specific equipment and skilled laboratory technicians, DAT is an effective and inexpensive technique for kala-azar detection in endemic regions. Nevertheless, regarding some disadvantages of DAT such as antigen fragility and cold chain requirement, it is recommended that researchers utilize both DAT and PCR combined together for appropriate diagnosis of visceral leishmaniasis. Ultimately, better identification of kala-azar entails more improved treatment plans and control strategies in the country. It is concluded that DAT and PCR are highly sensitive and specific to detect VL in rural endemic areas and they should be used as supplementary to each other. In animal subgroup, as reservoir hosts, different sensitivity and specificity of these tests were obtained, indicating their important role as screening tools in animal samples and valuable techniques in early detection of symptomatic and asymptomatic kala-azar patients.

\section{ACKNOWLEDGMENTS}

The authors thank Mr. Mohammad Aghapour for his kind assistance in proof editing and appreciate Ilam University of Medical Sciences, Ilam, Iran for financial support.

Declaration of Conflicting Interests: The authors declare that they have no conflict of interest.

Compliance with ethical standards: The study was approved by the Ilam University of Medical Sciences Ethics Committee and registered with NO: EC/92/H/132.

Funding source: This research was supported by Ilam University of Medical Sciences, Ilam, Iran (grant number 942008/71).

\section{REFERENCES}

1. Chappuis F, Sundar S, Hailu A, et al, Visceral leishmaniasis: what are the needs for diagnosis, treatment and control? Nature Rev Microbiol 2007; 5(11):873-882.

2. Motazedian M, Fakhar,M, Motazedian, MH, Hatam, G, Mikaeili F. A urine-based polymerase chain reaction method for the diagnosis of visceral leishmaniasis in immunocompetent patients. Diagn Microbiol Infect Dis 2008; 60(2): 151-154.

3. Badirzadeh A, Mohebali M, Ghasemian M, et al., Cutaneous and post kala-azar dermal leishmaniasis caused by Leishmania infantum in endemic areas of visceral leishmaniasis, northwestern Iran 20022011. Pathog Glob Health 2013; 107(4): 194-197.

4. Rakhshanpour A, Mohebali M, Akhondi B, Rahimi MT, Rokni MB. Serological Survey and Associated Risk Factors of Visceral Leish-maniasis in Qom Province, Central Iran. Iran J Public Health 2014; 43(1): 50-55. 
5. Abai MR, Oshaghi MA, Tajedin L, Rassi Y, Akhavan A. Geographical distribution and ecological features of the great gerbil subspecies in the main zoonotic cutaneous leishmaniasis foci in Iran. Asian Pac J Trop Biomed 2010; 3(10): 800-803.

6. Mniouil, $M$, Fellah $H$, Amarir $F$, et al, Epidemiological characteristics of visceral leishmaniasis in Morocco (1990-2014): an update. Acta Trop 2017; 170: 169-177.

7. Alborzi A, Pouladfar GR, Aelami MH. Visceral leishmaniasis; literature review and Iranian experience. Iran J Clin Infect Dis 2007; 2(2): 99-108.

8. Mohebali M. Visceral leishmaniasis in Iran: review of the epidemiological and clinical features. Iran $\mathrm{J}$ Parasitol 2013; 8(3):348-358.

9. Mohebali $M$, Hajjaran $H$, Hamzavi $Y$, et al, Epidemiological aspects of canine visceral leishmaniosis in the Islamic Republic of Iran. Vet Parasitol 2005; 129(3):243-251.

10. Desjeux P. Leishmaniasis: current situation and new perspectives. Comp Immunol Microbiol Infect Dis 2004; $27(5)$ : 305-318.

11. Fakhar M, E. Ahmad Pour, An Overview of theLaboratory Diagnostic Procedures of Visceral Leishmaniasis (Kala-Azar). Med Lab J 2013; 7(1): 45-54.

12. Khademvatan S, Mazloumi A, Fallah E, Jasem Saki. Detection of Leishmania infantum antigen in urinary samples by KAtex and comparison with serological methods. Ofogh-e-Danesh, Med J Gonabad Univ Med Sci 2011; 17: 6-20.

13. Davood R, Hejazi H, Ghasemi M. Evaluation and Comparison serological methods indirect immunofluorescence (IFAT) and direct agglutination (DAT) using standard antigen in the diagnosis of visceral leishmaniasis. Med J Mazandaran Univ Med Sci 2005; 15(49): 1-8.

14. Sakkas H, Constantina G, Levidiotou S. Laboratory diagnosis of human visceral leishmaniasis. J Vector Borne Dis 2016; 53(1): 8-16.

15. Baratloo A, Hosseini M, Negida A, El Ashal G. Part 1: Simple Definition and Calculation of Accuracy, Sensitivity and Specificity. Emerg (Tehran) 2015; 3(2): 48-49.

16. Moher D, Liberati A, Tetzlaff J, Altman DG. Preferred reporting items for systematic reviews and meta-analyses: the PRISMA statement. PLoS Med 2009; 6(7): e1000097.

17. Zijlstra E, Ali MS, El-hassam AM, et al, Kala-azar: a comparative study of parasitological methods and the direct agglutination test in diagnosis. Trans $R$ Soc Trop Med Hyg 1992; 86(5): 505-507.

18. Soleimanzadeh G, Edrissian GH, MovahhedDanes AM, Nadim A. Epidemiological aspects of kala-azar in Meshkin-Shahr, Iran: human infection. Bull World Health Organ 1993; 71(6): 759762.
19. Mohammadiha A, Haghighi A, Mohebali M, et al, Canine visceral leishmaniasis: a comparative study of real-time PCR, conventional PCR, and direct agglutination on sera for the detection of Leishmania infantum infection. Vet Parasitol 2013. 192 (1): 83-90.

20. Pandey K, Pandey BD, Mallik AK, et al. Diagnosis of visceral leishmaniasis by polymerase chain reaction of DNA extracted from Giemsa's solutionstained slides. J Parasitol Res 2010; 107(3):727730 .

21. moadab A, Alborzi A, Behbahani A, Shahyan jahromi F, Motazedian M. The early diagnosis of visceral leishmaniasis using peripheral blood and polymerase chain reaction. Sci J Hamdan Univ Med Sci 2003;16-21.

22. Silva ES, Schoone GJ, Gontijo CMF, Brazil RP, Pacheco RS, Schallig HDFH. Application of direct agglutination test (DAT) and fast agglutination screening test (FAST) for sero-diagnosis of visceral leishmaniasis in endemic area of Minas Gerais, Brazil. Kinetoplastid Biol Dis 2005; 4:4.

23. Chappuis F, Rijal S,Soto A, Menten J, Boelaert MA. Meta-analysis of the diagnostic performance of the direct agglutination test and rK39 dipstick for visceral leishmaniasis. BMJ 2006; 333(7571):723.

24. Gavgani ASM, Mohite H, Edrissian GH, Mohebali $\mathrm{M}$, Davies CR. Domestic dog ownership in Iran is a risk factor for human infection with Leishmania infantum. Am J Trop Med Hyg 2002; 67(5):511-515.

25. Mohammadi-Ghalehbin B, Hatam GR, Sarkari B, et al. A Leishmania infantum FML-ELISA for the detection of symptomatic and asymptomatic canine visceral leishmaniasis in an endemic area of Iran. Iran J immunol 2011; 8(4):244-250.

26. Mahami M, Mohebali M, Keshavarz H, Zareei Z. Comparison of direct agglutination test (DAT),indirect immunofluorescent antibody(IFAT)and Elisa in diagnosis of visceral Leishmaniasis.2008.

27. Imani $M$, Dehkharghani $A D$, Ghelman $M$, Mohammadloo M. Molecular technique for detection of Leishmania infantum isolates in Iran. Trop Parasitol 2014; 4(1):35-37.

28. Babakhan L, Mohebali M, Akhoundi B, Edrissian $\mathrm{GH}$, Keshavarz H. Rapid detection of Leishmania infantum infection in dogs: a comparative study using fast agglutination screening test (FAST) and direct agglutination test (DAT) in Iran. $\mathrm{J}$ Parasitol Res 2009; 105(3):717720.

29. Akhoundi B, Mohebali M, Babakhan L, et al. Rapid detection of human Leishmania infantum infection: A comparative field study using the fast agglutination screening test and the direct agglutination test. Travel Med Infect Dis. 2010; 8(5):305-310. 